\title{
The stability analysis of 3-RPR wind tunnel test bed based on workbench
}

\author{
Ziyan Shao, Wenjia Chen, Yongjin Hu, Guanjian Li \\ College of Mechanical Engineering, Yangzhou University, \\ Yangzhou, Jiangsu, China
}

Keywords: wind tunnel test bed, linear static structure analysis, modal analysis, harmonic response analysis

\begin{abstract}
This paper introduces a kind of wind tunnel test bed with a 3-RPR structure. Ansys workbench is chosen to be the main tool here to measure the structure's stability. Linear static structure analysis, modal analysis and harmonic response analysis are carried out to detect the force, the frequency and the mode of the structure. And this work would build a basis for later work on the wind tunnel test bed.
\end{abstract}

\section{Introduction}

Wind tunnel experiment is an essential stage of air vehicle development. Plenty of flow tests need to be carried out during the process of the development of each kind of the air vehicle. So the stability of the support structure has a crucial effect on the accuracy of the experiment results[1].

This paper introduces a kind of wind tunnel test bed based on 3-RPR structure with six degrees of freedom. It consists of a guide rail, 3-RPR planar parallel mechanisms, a bent-structure part, a test model and so on[1]. The organization of the structure is shown in figure1. The system can be a hard support in the experiment, realizing the motion of yawing, pitching and rolling of the model.

As a kind of engineering simulation technology platform, Ansys Workbench can provide a new project view function, combining the whole simulation process together, and completing the complex analysis by simple towing operation. This paper would conduct the stability analysis of the wind tunnel test bed[2] from three aspects: linear static structure analysis, modal analysis and harmonious response analysis. After removing unnecessary part of the system, the finial structure measured is shown in figure2

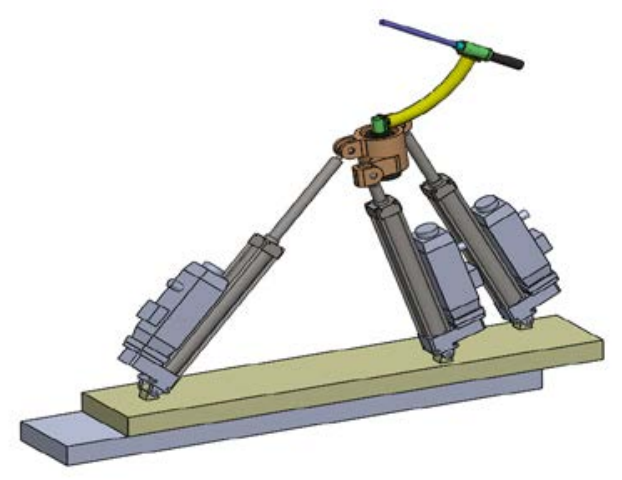

Figure1

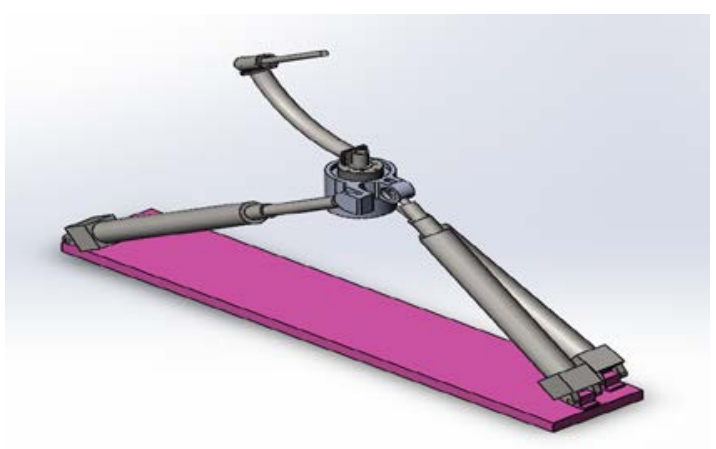

Figure2

\section{Linear Static Structure Analysis}

Linear static structure analysis[3] is the most basic and the most widely used type of analysis, usually applied to linear elastic materials and the condition of static loading. The so-called linear analysis has two meanings: first the material and the stress-strain relationship need to be linear and it can recover from the deformation; second the stiffness of the structure would not change when the structure has small displacement, small strain and small rotation. The static force means that the inertia and damping can be ignored when the structure is under the static load. At this time, the structure is in static equilibrium position. Regardless of the inertia, the quality has no effect on the structure[4]. 
The general equation based on classical force theory is shown as follows:

$$
[\mathrm{M}]\{\ddot{\mathrm{x}}\}+[\mathrm{C}]\{\dot{\mathrm{x}}\}+[\mathrm{K}]\{\mathrm{x}\}=\{\mathrm{F}(\mathrm{t})\}
$$

In the equation, $[\mathrm{M}]$ is mass matrix, $[\mathrm{C}]$ is damping matrix, $[\mathrm{K}]$ is stiffness matrix, $\{\mathrm{x}\}$ is displacement vector, $\{\mathrm{F}(\mathrm{x})\}$ is force vector, $\{\dot{\mathrm{x}}\}$ is velocity vector, and $\{\ddot{\mathrm{x}}\}$ is acceleration vector.

In the current structure analysis, quantities related with the time would be ignored, so the equation can be simplified as follow:

$$
[\mathrm{K}]\{\mathrm{x}\}=\{\mathrm{F}\}
$$

In the linear static structure analysis, the material properties only need the Young's modulus and the Poisson's ratio. After setting the material properties, we can add the fixed support to the base of the structure, carry out the mesh, and solve after adding the load. The deformation is shown in figure3, and the stress is shown in figure4.

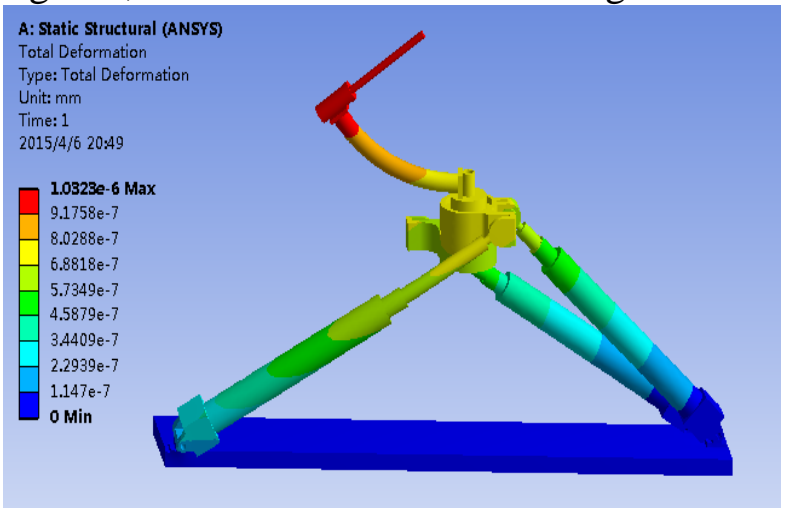

Figure3

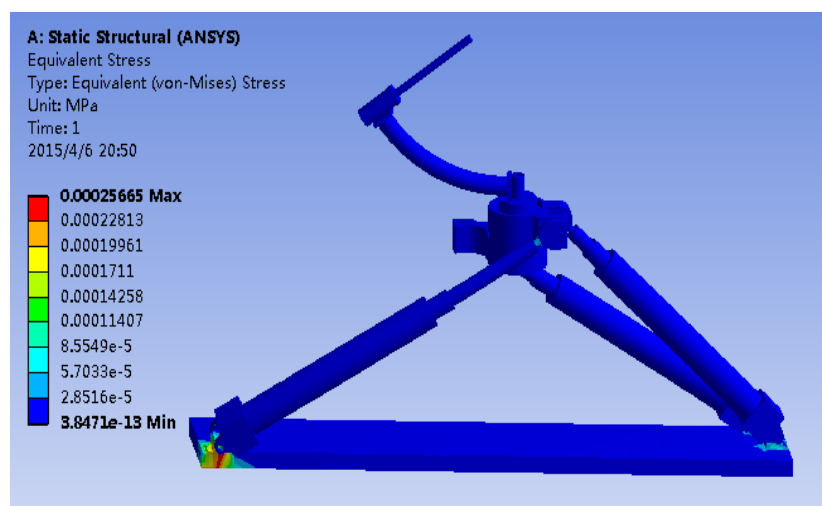

Figure4

Through the analysis we find that the maximum deformation occurs on the end-rolling-rod, and the maximum deformation is $1.03 \times 10-6 \mathrm{~mm}$. the maximum equivalent stress occurs on the base of the structure, and its value is $2.57 \times 10-4 \mathrm{MPa}$

\section{Modal Analysis}

Modal analysis[4] is a numerical technology calculating the structure vibration characteristic. The structure vibration characteristic includes natural frequency and vibration mode. Modal analysis is the most basic dynamics analysis as well as the basis of other dynamics analysis, which of extensive practical values. Modal analysis can help designers to avoid the resonance by determining the natural frequency vibration mode in the structure design.

The vibration frequency $\omega_{\mathrm{i}}$ and the modal $\emptyset_{\mathrm{i}}$ for the modal analysis is calculated out by the following equation:

$$
\left([\mathrm{K}]-\omega_{\mathrm{i}}^{2}[\mathrm{M}]\right)\left\{\varnothing_{\mathrm{i}}\right\}=0
$$

In this equation it is assumed that the stiffness matrix and the mass matrix are fixed. And the material needs to be linear elastic, using small displacement theory, undamped and has no exciting force[6].

The essential properties needed in the modal analysis are the Young's modulus, Poisson's ratio and density of the material. After adding fixed support to the base of the structure and solving the model, we get the first to the sixth modal, which is shown in table1.

Table1 Results of Modal Analysis

\begin{tabular}{|c|c|}
\hline First mode & Second mode \\
Frequency: $24.549 \mathrm{~Hz}$ & Frequency: $36.924 \mathrm{~Hz}$ \\
Maximum deformation:6.351mm & Maximum deformation: $11.222 \mathrm{~mm}$ \\
\hline
\end{tabular}




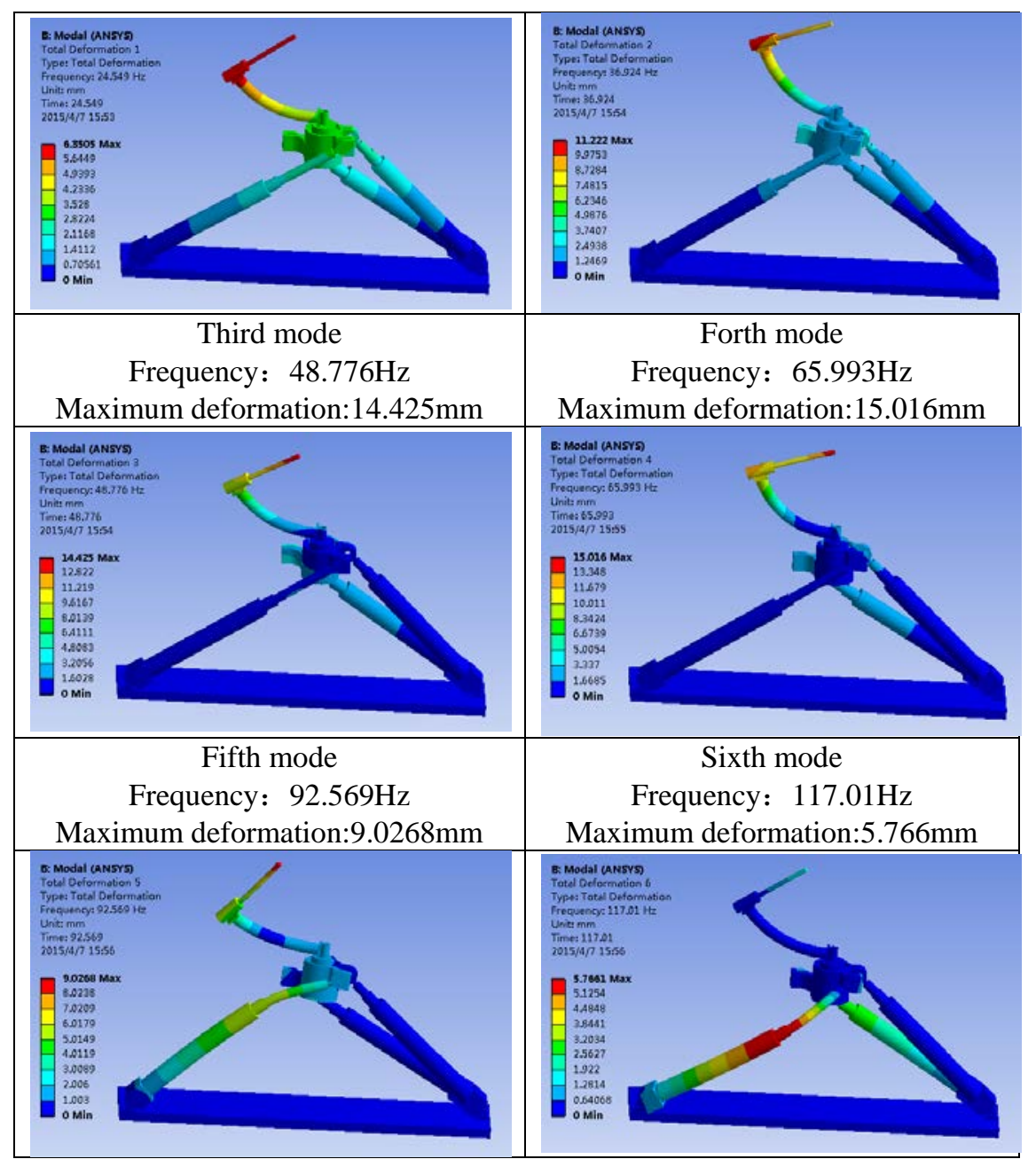

From the analysis and the result we find that the first to the third modes are more stable than the other three modes. So we choose the frequency which range from $20 \mathrm{~Hz}$ to $70 \mathrm{~Hz}$ to do the harmonic response analysis.

\section{Harmonic Response Analysis}

Harmonic response analysis[4] is a kind of time-domain analysis. It is used to calculate the time course of the structure response. But it only confines to the steady forced vibration of the structure where the loading has simple harmonic changes regardless of the transient oscillation which is loaded at start. Harmonic response analysis can carry out sweep frequency analysis, analysing the response of the structure under different simple harmonic load with different frequency and amplitude in order to detect the resonance. As a result, it can help the designer to avoid structural resonance so as to ensure that the structure can withstand various harmonic loadings with different frequency.

The loading of harmonic response analysis is changing with time by sine model. It can calculate the structure response caused by a series of different loadings with different frequency and amplitude. The equation of harmonic response analysis is shown as follows

$$
[\mathrm{M}]\{\ddot{\mathrm{x}}\}+[\mathrm{C}]\{\dot{\mathrm{x}}\}+[\mathrm{K}]\{\mathrm{x}\}=\left\{\mathrm{F}_{0} \cos (\omega \mathrm{t})\right\}
$$

In the equation, $[\mathrm{M}]$ is mass matrix, [C] is damping matrix, $[\mathrm{K}]$ is stiffness matrix, $\{\mathrm{x}\}$ is displacement vector, $\{\mathrm{F}(\mathrm{x})\}$ is force vector, $\{\dot{\mathrm{x}}\}$ is velocity vector, and $\{\ddot{\mathrm{x}}\}$ is acceleration vector[7].

After setting material properties, adding the fixed support and the loading, solve the model and get the curve as is shown in table2.

We get stress frequency response and deformation frequency response from the analysis. We can figure out that when the frequency is $47 \mathrm{~Hz}$, the stability of the structure is the worst. So $47 \mathrm{~Hz}$ is 
used in the following step as the frequency to calculate the deformation and the stress. And the result is shown in table3

Table 2 Results of Harmonic Response Analysis

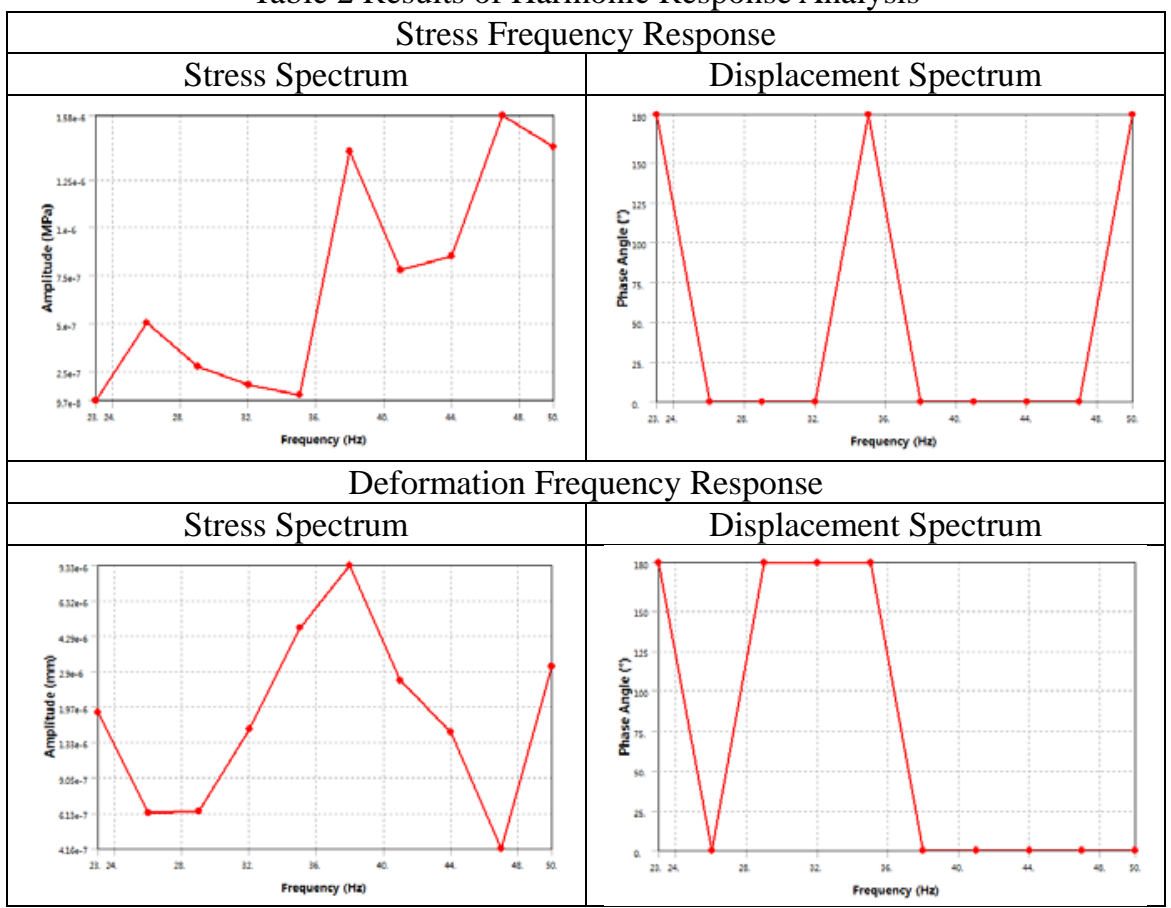

Table 3 Equivalent Stress and Equivalent Deformation

\begin{tabular}{|c|c|}
\hline $\begin{array}{c}\text { Equivalent Stress } \\
\text { Min: } 5.10 \times 10^{-14} \mathrm{MPa} \\
\text { Max: } 2.83 \times 10^{-3} \mathrm{MPa}\end{array}$ & 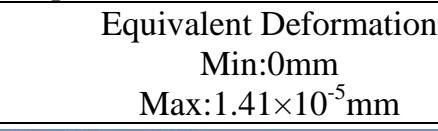 \\
\hline 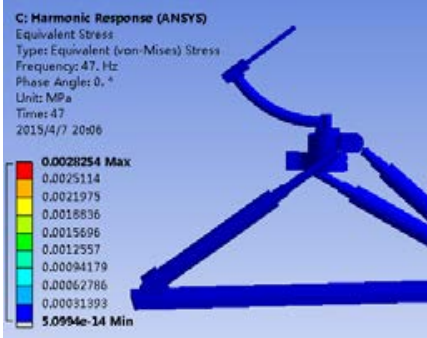 & 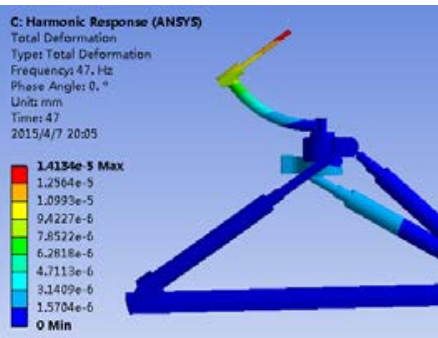 \\
\hline
\end{tabular}

\section{Conclusions}

After carrying out the analysis in workbench, plenty of data and curves about the structure are obtained. These results could not only help the designers to understand the structure better but also keep the designers from using the improper frequency. Meanwhile, the work can provide theoretical support for the future research.

\section{Acknowledgements}

The authors would like to acknowledge the financial support of the Nature Science Foundation of Jiangsu Province of China (No.BK2012687) and the National Natural Science Foundation of China (No.50975249).

\section{References}

[1] Jian Zhang, Kinematics modeling research on wind tunnel test bed with mixed and multi-degree of freedom based on 3-RPR structure. Yangzhou, 2012. In Chinese. 
[2] Bing Li, Zhengjia He, Xuefeng Chen, Design, Simulation and Optimization of ANSYS Workbench, Tsinghua University Press: Beijing, 2011. In Chinese.

[3] Guilong Ling, Jinbin Ding, Zheng Wen, ANSYS Workbench 13.0 from introduction to master, Tsinghua University Press: Beijing, 2012. In Chinese.

[4] Zhixin Huang, Chengzhu Liu, the study book of Ansys Workbench 14, POSTS\&TELECOM PRESS, Beijing, 2013. In Chinese.

[5] Mingbo Shao, The Fluid-Solid Interaction Analysis of An Airfoil Based on ANSYS, Harbin,2012. In Chinese.

[6] Xijian Zheng, Zhengyi Xie, Guozhong Zhang, Construction Machinery, 2007(21). In Chinese.

[7] Zhimin Yang, Jian Zhou, Lijun Li, Xueliang Cheng, Journal of Agricultural Mechanization Research, 2013(12). In Chinese. 